\title{
HIGH-TEMPERATURE FLUOROCARBON CHEMISTRY REVISITED
}

\author{
C. J. Cobos ${ }^{1}$, K. Hintzer ${ }^{2}$, L. Sölter ${ }^{3,4}$, E. Tellbach ${ }^{3,4}$, \\ A. Thaler, ${ }^{2}$ and J. Troe $3,4, *$
}

Supporting Information

\footnotetext{
${ }^{1}$ INIFTA, Facultad de Ciencias Exactas, Universidad Nacional de La Plata, CONICET, Argentina

${ }^{2}$ Dyneon GmbH, Gendorf, D-84504 Burgkirchen, Germany

${ }^{3}$ Institut für Physikalische Chemie, Universität Göttingen, Tammannstrasse 6, D-37077 Göttingen, Germany

${ }^{4}$ Max-Planck-Institut für Biophysikalische Chemie, Am Fassberg 11, D-37077 Göttingen, Germany

* Email: juergen.troe@mpibpc.mpg.de
} 


\section{SI - I Modelling of oscillator strengths for near - UV absorptions}

Table S1. Oscillator strengths f and maximum absorption cross sections $\sigma_{\max }(T)$.

Oscillator strengths were determined using time-dependent density functional theory (TD-

DFT) on the $\omega$ B97X-D level (for a comparison with results obtained using other

functionals, see e. g. ref. $\mathrm{S} 1 ; \lambda_{\max }=$ position of the band maximum, $\mathrm{f}=$ oscillator strength; $\sigma_{\max }(T)=$ experimental absorption cross section at $\lambda_{\max }$ and $T(\mathrm{~K})$, extrapolated if $\lambda_{\max }<$ $200 \mathrm{~nm}$; references given below: th $=$ theoretical, $\exp =$ experimental, $\mathrm{pw}=$ present work).

\begin{tabular}{|c|c|c|c|c|}
\hline Molecule & $\lambda_{\max } / \mathrm{nm}$ & $\mathrm{f}$ & $\sigma_{\max }(T) / 10^{-18} \mathrm{~cm}^{2}$ & ref. \\
\hline$\overline{\mathrm{C}_{2} \mathrm{~F}_{4}}$ & 193 & 0.0225 & & $\mathrm{~S} 2$ (th) \\
\hline & 193 & & $5.3(298)$ & $\mathrm{S} 3(\exp )$ \\
\hline $\mathrm{C}_{2} \mathrm{~F}_{2}$ & 177 & 0.111 & & $\mathrm{~S} 2$ (th) \\
\hline \multirow[t]{3}{*}{$\mathrm{C}_{2} \mathrm{~F}$} & 235 & 0.0048 & & $\mathrm{~S} 2(\mathrm{th})$ \\
\hline & 205 & 0.0176 & & $\mathrm{~S} 2$ (th) \\
\hline & 172 & 0.0512 & & S2(th) \\
\hline \multirow[t]{2}{*}{$\overline{\mathrm{CF}_{2}}$} & 256 & 0.0348 & & $\mathrm{~S} 2$ (th) \\
\hline & 248 & & $4.5(1500)$ & $\mathrm{pw}(\exp )$ \\
\hline \multirow[t]{4}{*}{$\mathrm{CF}$} & 222 & 0.0259 & & S2(th) \\
\hline & 215 & & $6.6(3220)$ & pw(exp) \\
\hline & 197 & 0.0483 & & S2(th) \\
\hline & 200 & & $8.1(3220)$ & pw(exp) \\
\hline \multirow[t]{3}{*}{$\mathrm{C}_{2}$} & 332 & 0.0588 & & pw(th) \\
\hline & 350 & & $0.5(3340)$ & pw(exp) \\
\hline & 196 & 0.0009 & & pw(th) \\
\hline & 137 & 0.0939 & & pw(th) \\
\hline & 200 & & $6.6(3810)$ & pw(exp) \\
\hline
\end{tabular}




\section{SI - II Modelling of rate constants}

Rate and equilibrium constants have been modelled with the methodology used before and described, for example, in the ESI of ref. S2 and in ref. S4. Optimized structures and vibrational frequencies were derived with the B3LYP and M06-2X functionals combined with the $6-311+\mathrm{G}(3 \mathrm{df})$ basis set for $\mathrm{C}$ and $\mathrm{F}$ atoms, characterizing the potential energy surface of dissociating molecules. Thermochemistry data and Morse parameters $\beta$ were obtained from single-point G4//B3LYP/6-311+G(3df) and G4//M06-2X/6-311+G(3df) calculations.

a. Falloff curves for the reaction $\mathrm{CF}_{2}(+\mathrm{Ar}) \rightarrow \mathrm{CF}+\mathrm{F}(+\mathrm{Ar})$

Electronic potential along the minimum energy path (MEP)

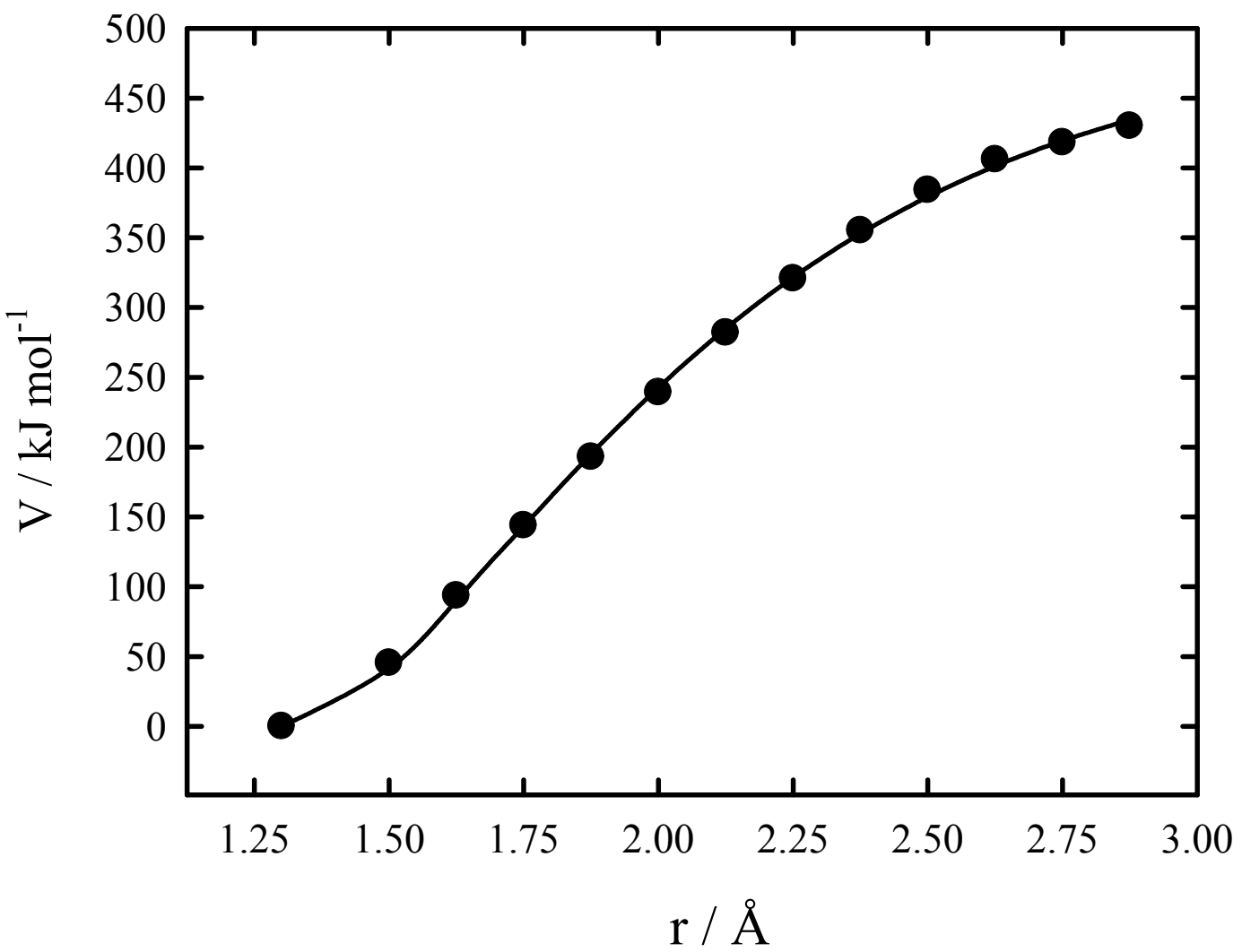

Fig. S1. Electronic potential for $\mathrm{CF}_{2} \rightarrow \mathrm{CF}+\mathrm{F}$ calculated at the G4//B3LYP/6-311+G(3df) level. Solid line: fit with a Morse function $\left(\beta=1.71 \AA^{-1}\right)$. 


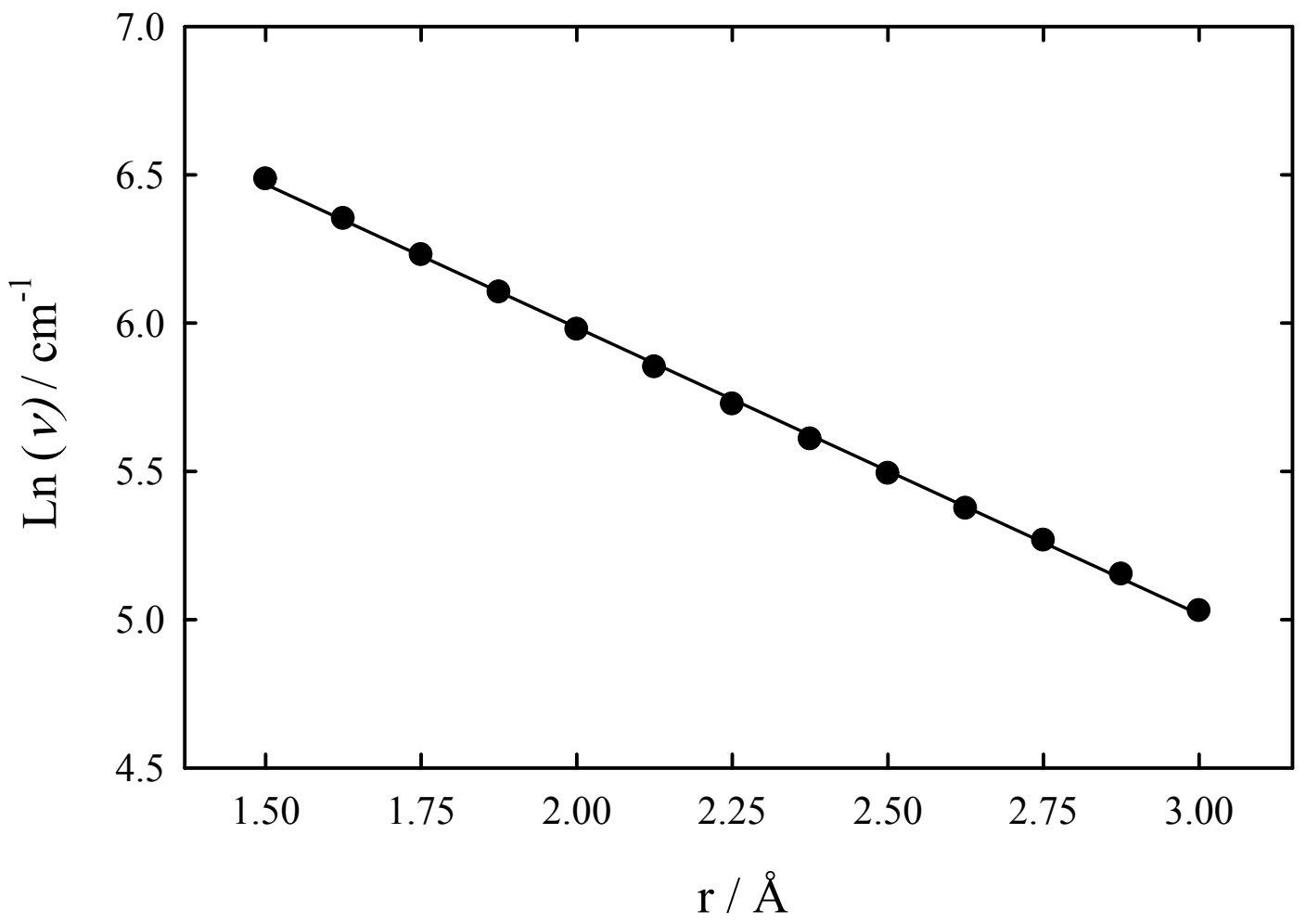

Fig. S2. Frequency of the $\mathrm{CF}_{2}$ bending mode along the MEP, calculated at the B3LYP/6$311+\mathrm{G}(3 \mathrm{df})$ level. Solid line: fit with an exponential decay function $\left(\alpha=0.97 \AA^{-1}\right)$. 


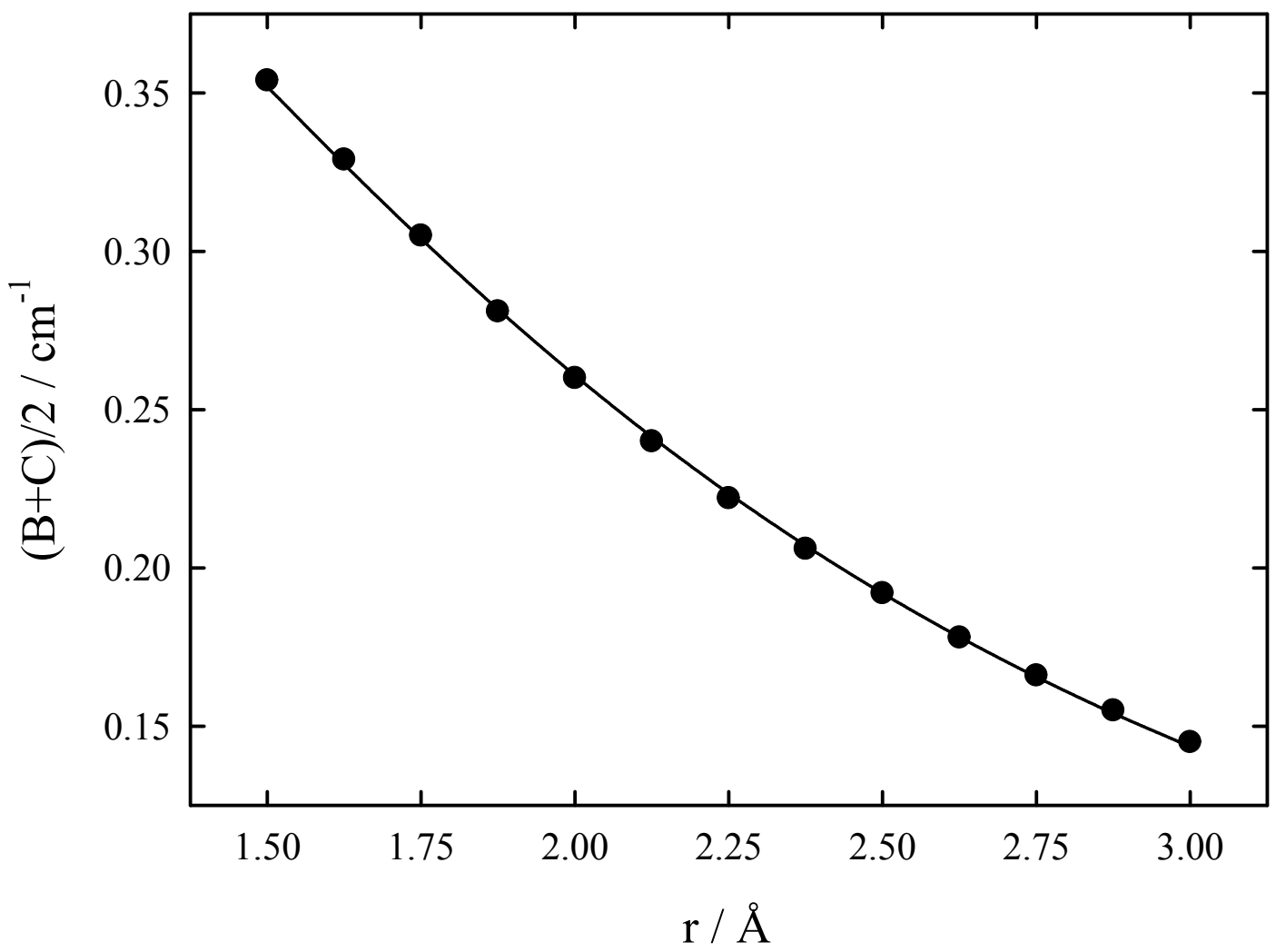

Fig.

Fig. S3. Rotational constants of $\mathrm{CF}_{2}$ along the MEP calculated at the B3LYP/6-311+G(3df) level. Solid line: fit with $(\mathrm{B}+\mathrm{C}) / 2=0.392 \mathrm{~cm}^{-1} /\left[1+0.511(\mathrm{r}-1.301)+0.299(\mathrm{r}-1.301)^{2}\right]$. 


\section{Calculation of the limiting high-pressure rate constant $k_{1, \infty}$}

Table S2. High-pressure rate constants for $\mathrm{CF}+\mathrm{F} \rightarrow \mathrm{CF}_{2}\left(\right.$ in $\left.^{3} \mathrm{~cm}^{3} \mathrm{~mol}^{-1} \mathrm{~s}^{-1}\right)$ and $\mathrm{CF}_{2} \rightarrow \mathrm{CF}+\mathrm{F}$ (in $\mathrm{s}^{-1}$ ). SACM/CT calculations with the parameters $v=1.32$ and $\mathrm{C}_{v}=2.47 \times 10^{-3} \mathrm{~cm}^{-1}$, for details see ref. S4 (PST = phase space theory).

\begin{tabular}{|c|c|c|c|c|}
\hline$T / \mathrm{K}$ & $k_{2, \infty}(\mathrm{PST})$ & $\mathrm{f}_{\text {rigid }}$ & $k_{2, \infty}$ & $k_{1, \infty}$ \\
\hline 2500 & $2.35 \times 10^{13}$ & 0.776 & $1.83 \times 10^{13}$ & $2.54 \times 10^{4}$ \\
\hline 3500 & $2.49 \times 10^{13}$ & 0.746 & $1.86 \times 10^{13}$ & $2.34 \times 10^{7}$ \\
\hline 4500 & $2.53 \times 10^{13}$ & 0.721 & $1.82 \times 10^{13}$ & $9.85 \times 10^{8}$ \\
\hline
\end{tabular}

$k_{1, \infty}=1.24 \times 10^{15}(T / 3500 \mathrm{~K})^{-0.84} \exp (-62245 \mathrm{~K} / T) \mathrm{s}^{-1}$

\section{Calculation of limiting low-pressure rate constants $k_{1,0}$}

Table S3. Low-pressure rate constants for $\mathrm{CF}_{2}(+\mathrm{Ar}) \rightarrow \mathrm{CF}+\mathrm{F}(+\mathrm{Ar})\left(\right.$ in $\left.\mathrm{cm}^{3} \mathrm{~mol}^{-1} \mathrm{~s}^{-1}\right)$. $\mathrm{F}_{\text {rot }}$ calculated with the potential of Fig. 1 and the rotational constants of Fig. 3. As in our previous articles, the $\beta_{\mathrm{c}}$ values were calculated using (for $\mathrm{M}=\mathrm{Ar}$ ) a temperature independent value of $<\Delta \mathrm{E} / \mathrm{hc}>=100 \mathrm{~cm}^{-1}$ (superscript $\mathrm{SC}=$ strong collision rate constant).

\begin{tabular}{|c|c|c|c|}
\hline$T / \mathrm{K}$ & $k_{0} \mathrm{SC}$ & $\beta_{\mathrm{c}}$ & $k_{0}$ \\
\hline 2500 & $4.51 \times 10^{7}$ & 0.042 & $1.90 \times 10^{6}$ \\
\hline 3500 & $2.57 \times 10^{10}$ & 0.030 & $7.71 \times 10^{8}$ \\
\hline 4500 & $8.03 \times 10^{11}$ & 0.023 & $1.85 \times 10^{10}$ \\
\hline
\end{tabular}

$$
\begin{aligned}
& k_{1,0} \mathrm{SC}=[\mathrm{Ar}] 6.14 \times 10^{17}(T / 3500 \mathrm{~K})^{-1.33} \exp (-59465 \mathrm{~K} / T) \quad \mathrm{cm}^{3} \mathrm{~mol}^{-1} \mathrm{~s}^{-1} \\
& k_{1,0}=[\mathrm{Ar}] 2.07 \times 10^{16}(T / 3500 \mathrm{~K})^{-2.48} \exp (-59880 \mathrm{~K} / T) \quad \mathrm{cm}^{3} \mathrm{~mol}^{-1} \mathrm{~s}^{-1}
\end{aligned}
$$




\section{Calculation of falloff curves}

Falloff curves were calculated employing the model of ref. S5 with $\mathrm{F}_{\text {cent }}=\mathrm{F}_{\text {cent }}{ }^{\mathrm{SC}} \times \mathrm{F}_{\text {cent }}{ }^{\mathrm{WC}}=$ $\mathrm{F}_{\text {cent }}{ }^{\mathrm{SC}} \mathrm{X} 0.64=0.36(2500 \mathrm{~K}), 0.33(3500 \mathrm{~K})$ and $0.32(4500 \mathrm{~K})$.

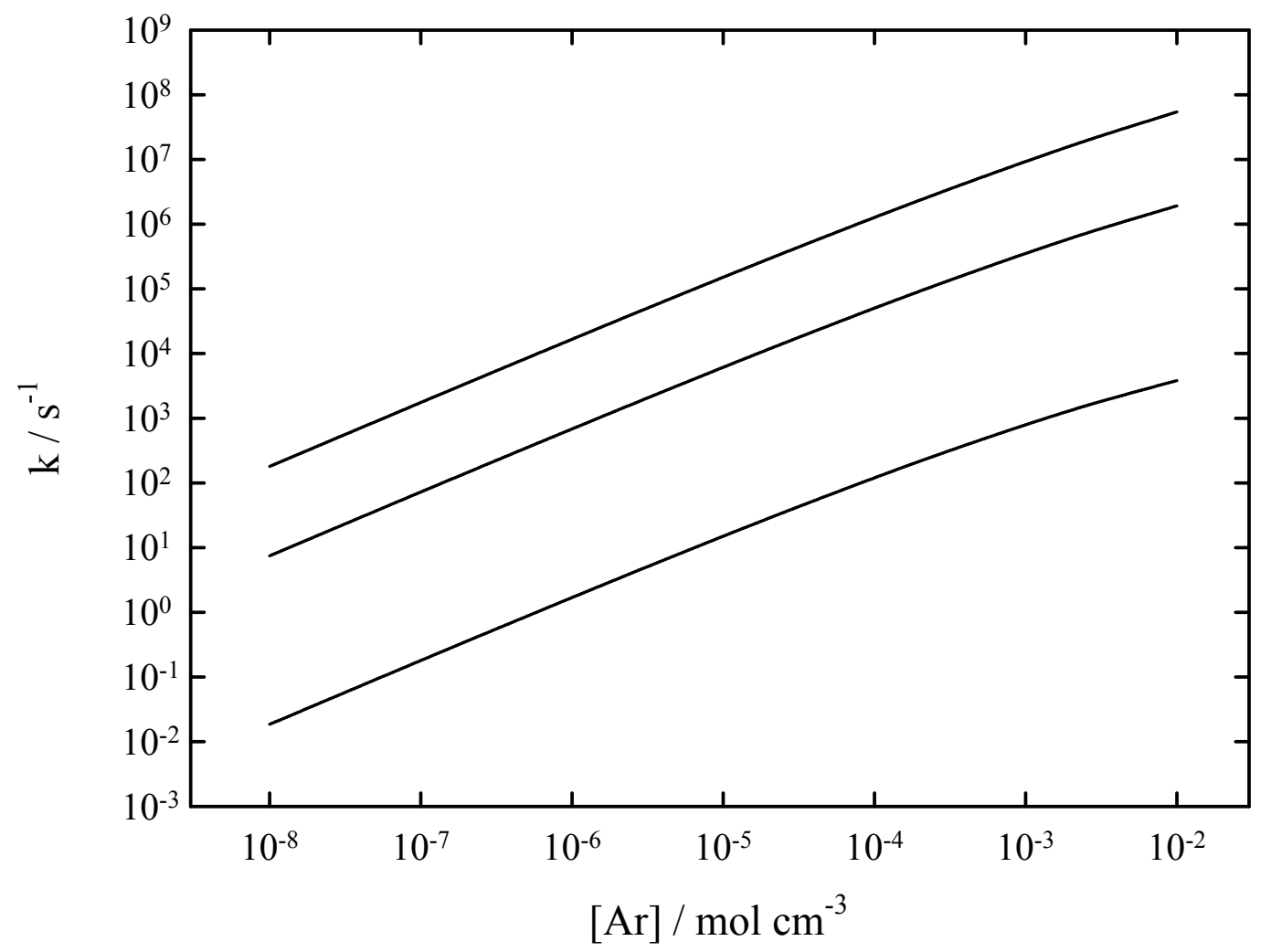

Fig.

Fig. S4. Falloff curves for the reaction $\mathrm{CF}_{2}(+\mathrm{Ar}) \rightarrow \mathrm{CF}+\mathrm{F}(+\mathrm{Ar})$. Calculations for 2500,3500 and $4500 \mathrm{~K}$ (from bottom to top).

\section{Molecular parameters}

Bond dissociation enthalpy for $\mathrm{CF}_{2} \rightarrow \mathrm{CF}+\mathrm{F}$ at $0 \mathrm{~K}: 510.4 \mathrm{~kJ} \mathrm{~mol}^{-1}(\mathrm{~S} 6)$.

$\mathrm{CF}_{2}$ vibrational frequencies: $666.25,1114.44$, and $1225.08 \mathrm{~cm}^{-1}(\mathrm{~S} 7, \mathrm{~S} 8)$.

$\mathrm{CF}_{2}$ rotational constants: $2.951,0.420$, and $0.368 \mathrm{~cm}^{-1}(\sigma=2)(\mathrm{S} 9)$.

CF vibrational frequency: $1308.1 \mathrm{~cm}^{-1}$.

CF rotational constant: $1.4172 \mathrm{~cm}^{-1}$.

$<\Delta \mathrm{E}>/ \mathrm{hc}=-100 \mathrm{~cm}^{-1}$ assumed for the calculation of $k_{1,0}$. 
b. Falloff curves for the reaction $\mathrm{C}_{2} \mathrm{~F}(+\mathrm{Ar}) \rightarrow \mathrm{C}_{2}+\mathrm{F}(+\mathrm{Ar})$

Electronic potential along the MEP

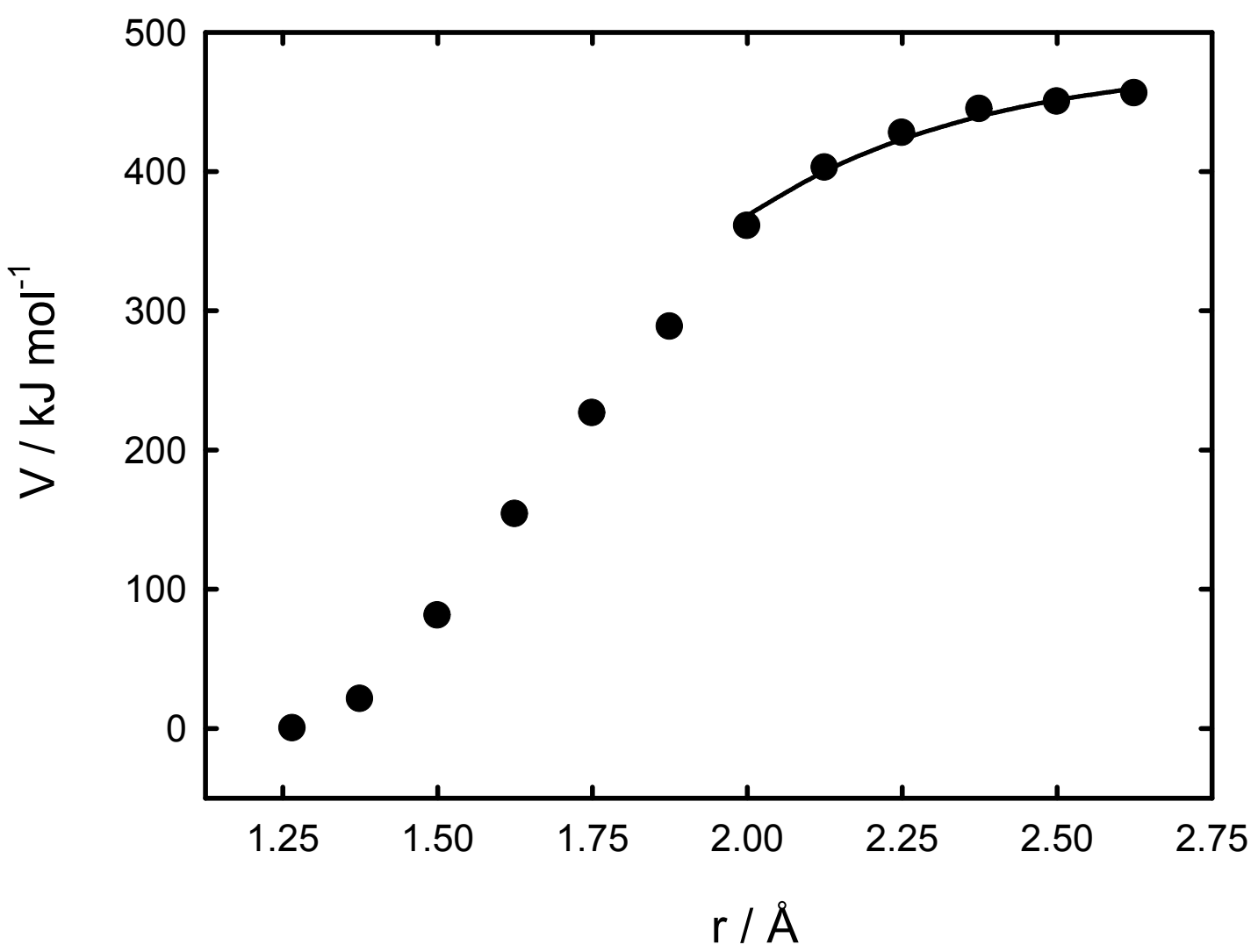

Fig. S5. Electronic potential for $\mathrm{C}_{2} \mathrm{~F} \rightarrow \mathrm{C}_{2}+\mathrm{F}$ calculated at the $\mathrm{G} 4 / \mathrm{B} 3 \mathrm{LYP} / 6-311+\mathrm{G}(3 \mathrm{df})$ level. Solid line: fit with a Morse function $\left(\beta=2.85 \AA^{-1}\right)$. 


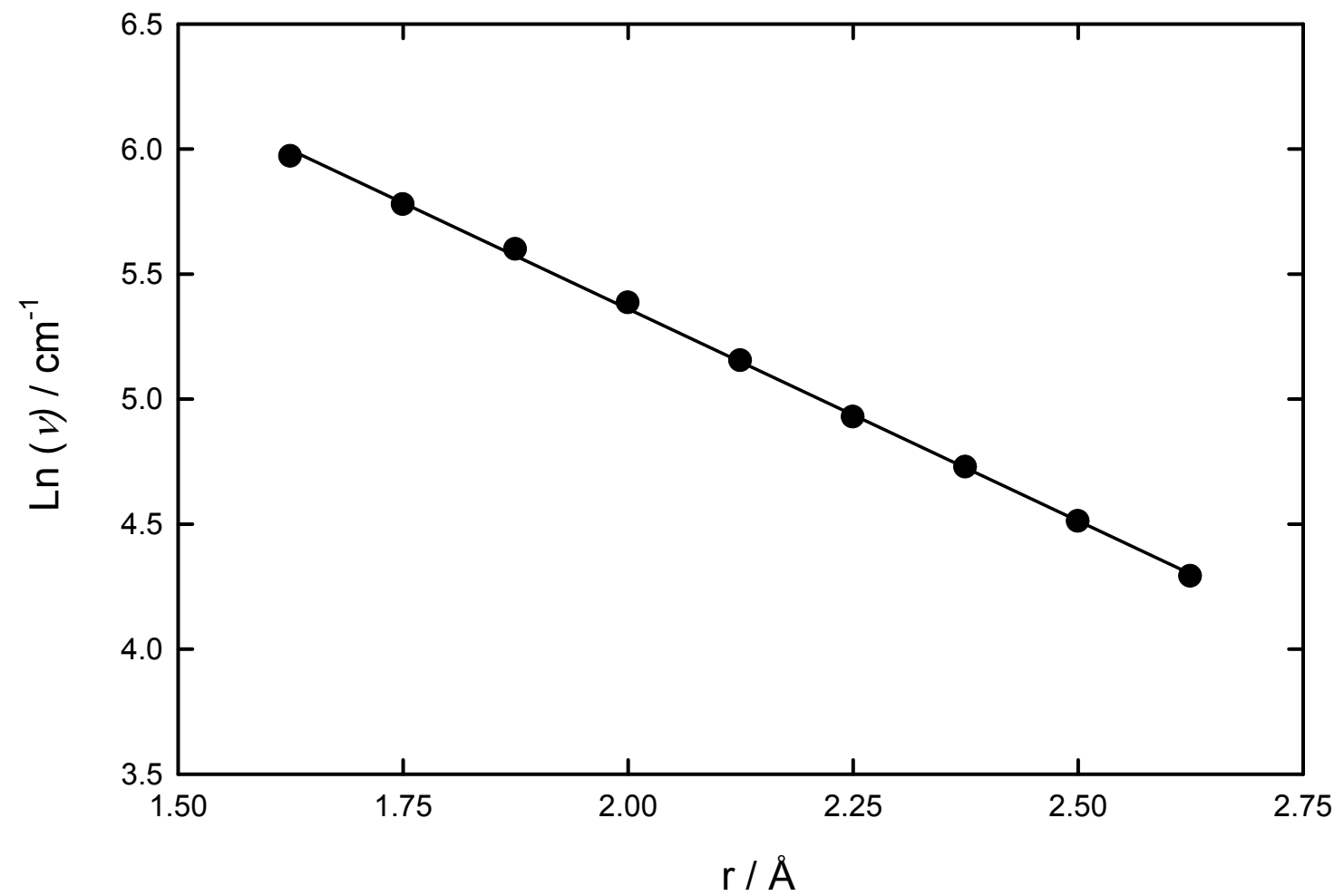

Fig. S6. Frequency of the $\mathrm{C}_{2} \mathrm{~F}$ bending mode along the MEP, calculated at the B3LYP/6$311+\mathrm{G}(3 \mathrm{df})$ level. Solid line: fit with an exponential decay function $\left(\alpha=1.69 \AA^{-1}\right)$. 


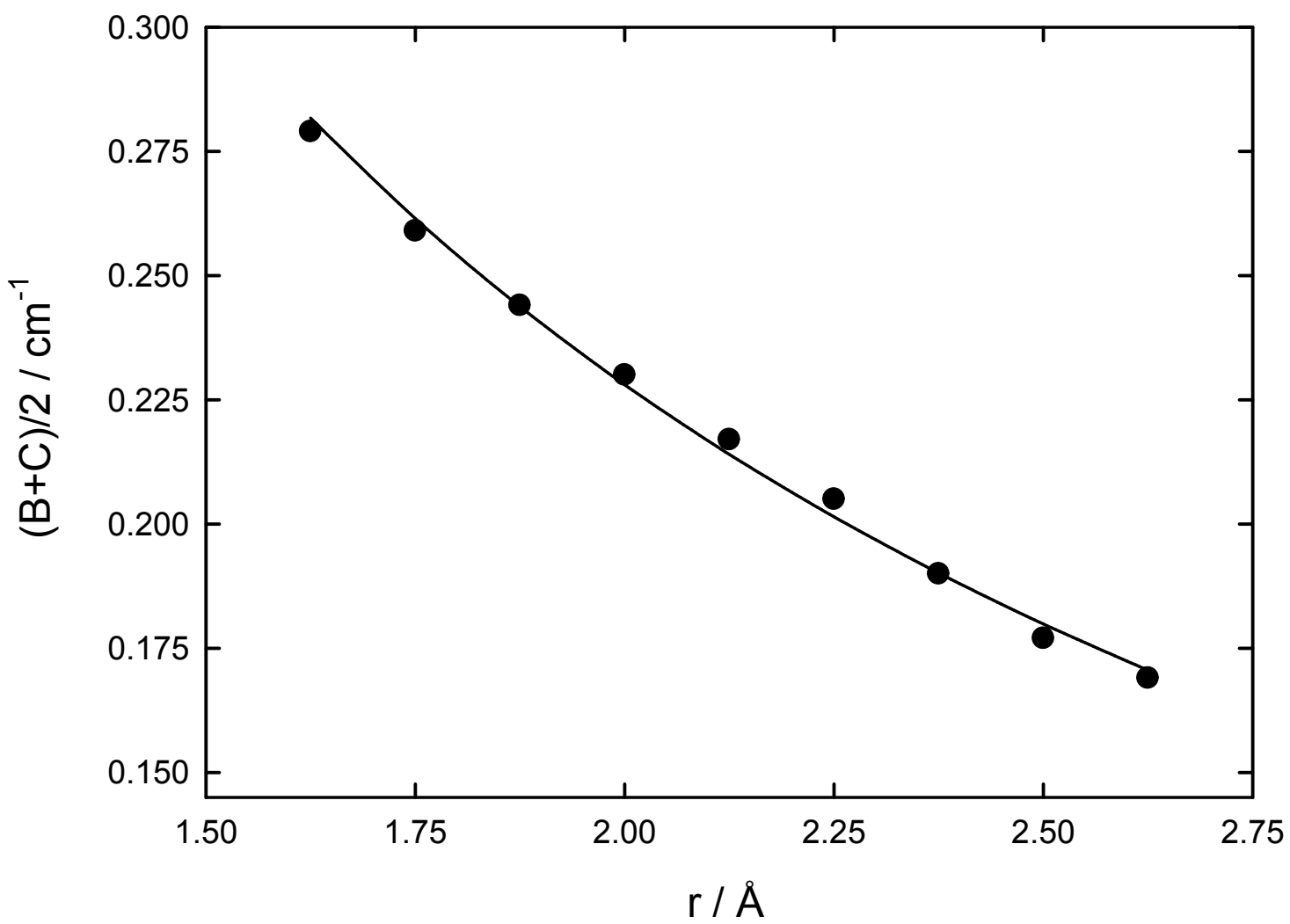

Fig. S7. Rotational constants of $\mathrm{C}_{2} \mathrm{~F}$ along the MEP, calculated at the B3LYP/6-311+G(3df) level. Solid line: fit with the function $(B+C) / 2=0.359 \mathrm{~cm}^{-1} /[1+0.747(\mathrm{r}-1.266)+0.0487(\mathrm{r}-$ $\left.1.266)^{2}\right]$.

\section{Calculation of $\boldsymbol{k}_{8, \infty}$}

Table S4. High-pressure rate constants for $\mathrm{C}_{2}+\mathrm{F} \rightarrow \mathrm{C}_{2} \mathrm{~F}\left(\right.$ in $\mathrm{cm}^{3} \mathrm{~mol}^{-1} \mathrm{~s}^{-1}$ ) and $\mathrm{C}_{2} \mathrm{~F} \rightarrow \mathrm{C}_{2}+\mathrm{F}$ (in $\mathrm{s}^{-1}$ ). SACM/CT calculations with the parameters $v=1.13$ and $\mathrm{C}_{v}=3.25 \times 10^{-2} \mathrm{~cm}^{-1}$, for details see ref. S4.

\begin{tabular}{|c|c|c|c|c|}
\hline$T / \mathrm{K}$ & $k_{-8, \infty}(\mathrm{PST})$ & $\mathrm{f}_{\text {rigid }}$ & $k_{-8, \infty}$ & $k_{8, \infty}$ \\
\hline 2500 & $2.28 \times 10^{14}$ & 0.278 & $6.32 \times 10^{13}$ & $9.65 \times 10^{4}$ \\
\hline 3500 & $2.48 \times 10^{14}$ & 0.267 & $6.63 \times 10^{13}$ & $3.69 \times 10^{7}$ \\
\hline 4500 & $2.63 \times 10^{14}$ & 0.259 & $6.81 \times 10^{13}$ & $6.43 \times 10^{8}$ \\
\hline
\end{tabular}

$$
k_{8, \infty}=2.36 \times 10^{14}(T / 3500 \mathrm{~K})^{-0.961} \exp (-54855 \mathrm{~K} / T) \mathrm{s}^{-1}
$$




\section{Calculation of $\boldsymbol{k}_{\mathbf{8}, 0}$}

Table S5. Low-pressure rate constants for $\mathrm{C}_{2} \mathrm{~F}(+\mathrm{Ar}) \rightarrow \mathrm{C}_{2}+\mathrm{F}(+\mathrm{Ar})\left(\mathrm{cm}^{3} \mathrm{~mol}^{-1} \mathrm{~s}^{-1}\right) \cdot \beta_{\mathrm{c}}$ values were calculated using a temperature independent value of $-<\Delta \mathrm{E}>=100 \mathrm{~cm}^{-1}$.

\begin{tabular}{|c|c|c|c|}
\hline$T / \mathrm{K}$ & $k_{8,0} \mathrm{SC}$ & $\beta_{\mathrm{c}}$ & $k_{8,0}$ \\
\hline 2500 & $3.62 \times 10^{8}$ & 0.042 & $1.52 \times 10^{7}$ \\
\hline 3500 & $8.70 \times 10^{10}$ & 0.030 & $2.61 \times 10^{9}$ \\
\hline 4500 & $1.53 \times 10^{12}$ & 0.023 & $3.51 \times 10^{10}$ \\
\hline
\end{tabular}

$$
k_{8,0}=[\operatorname{Ar}] 2.98 \times 10^{16}(T / 3500 \mathrm{~K})^{-4.02} \exp (-56880 \mathrm{~K} / T) \quad \mathrm{cm}^{3} \mathrm{~mol}^{-1} \mathrm{~s}^{-1}
$$

\section{Calculation of falloff curves}

Falloff curves were calculated employing the model of ref. S5 with $\mathrm{F}_{\text {cent }}=\mathrm{F}_{\text {cent }}{ }^{\mathrm{SC}} \times \mathrm{F}_{\text {cent }}{ }^{\mathrm{WC}}=$ $\mathrm{F}_{\text {cent }}{ }^{\mathrm{SC}} \mathrm{x} 0.64=0.36(2500 \mathrm{~K}), 0.34(3500 \mathrm{~K})$ and $0.33(4500 \mathrm{~K})$ values.

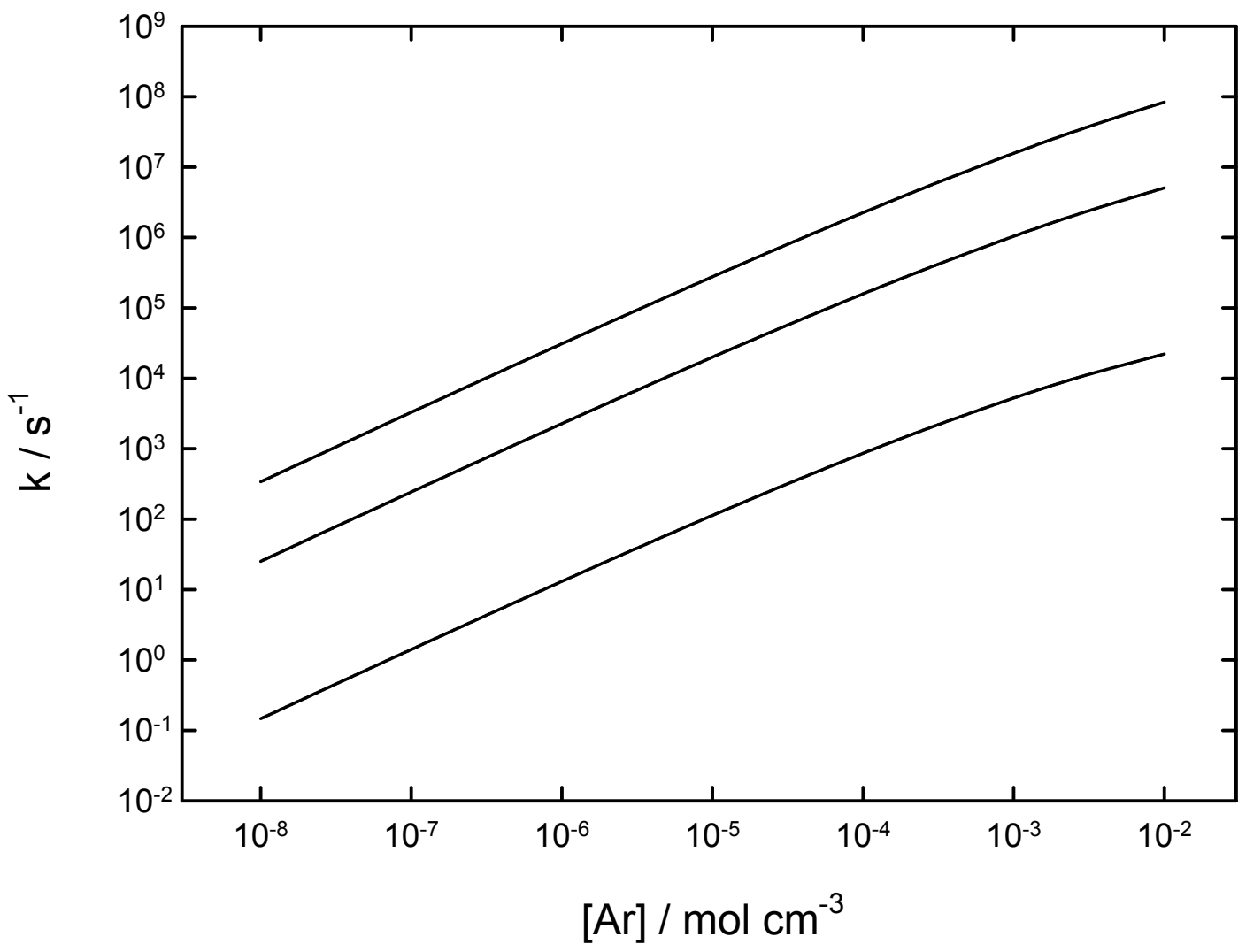

Fig. S8. Falloff curves for the reaction $\mathrm{C}_{2} \mathrm{~F}(+\mathrm{Ar}) \rightarrow \mathrm{C}_{2}+\mathrm{F}(+\mathrm{Ar})$. Calculations for 2500,3500 and $4500 \mathrm{~K}$ (from bottom to top). 


\section{Molecular parameters}

Bond dissociation enthalpy for $\mathrm{C}_{2} \mathrm{~F} \rightarrow \mathrm{C}_{2}+\mathrm{F}$ at $0 \mathrm{~K}: 444.8 \mathrm{~kJ} \mathrm{~mol}^{-1}$ (from G4//B3LYP/6$311+\mathrm{G}(3 \mathrm{df})$ calculations).

$\mathrm{C}_{2}$ Fvibrational frequencies: $370,1018,1819 \mathrm{~cm}^{-1}$ (from B3LYP/6-311+G(3df) calculations).

$\mathrm{C}_{2} \mathrm{~F}$ rotational constants: 50.041, $0.359(2) \mathrm{cm}^{-1}(\sigma=1)$ (from B3LYP/6-311+G(3df) calculations).

$\mathrm{C}_{2}$ vibrational frequency: $1873 \mathrm{~cm}^{-1}$ (from B3LYP/6-311+G(3df) calculations).

$\mathrm{C}_{2}$ rotational constants: $1.807 \mathrm{~cm}^{-1}(\sigma=2)$ (from B3LYP/6-311+G(3df) calculations). 


\section{c. Falloff curves for the reaction $\mathrm{C}_{2} \mathrm{~F}_{2}(+\mathrm{Ar}) \rightarrow \mathrm{C}_{2} \mathrm{~F}+\mathrm{F}(+\mathrm{Ar})$}

\section{Electronic potential along the MEP}

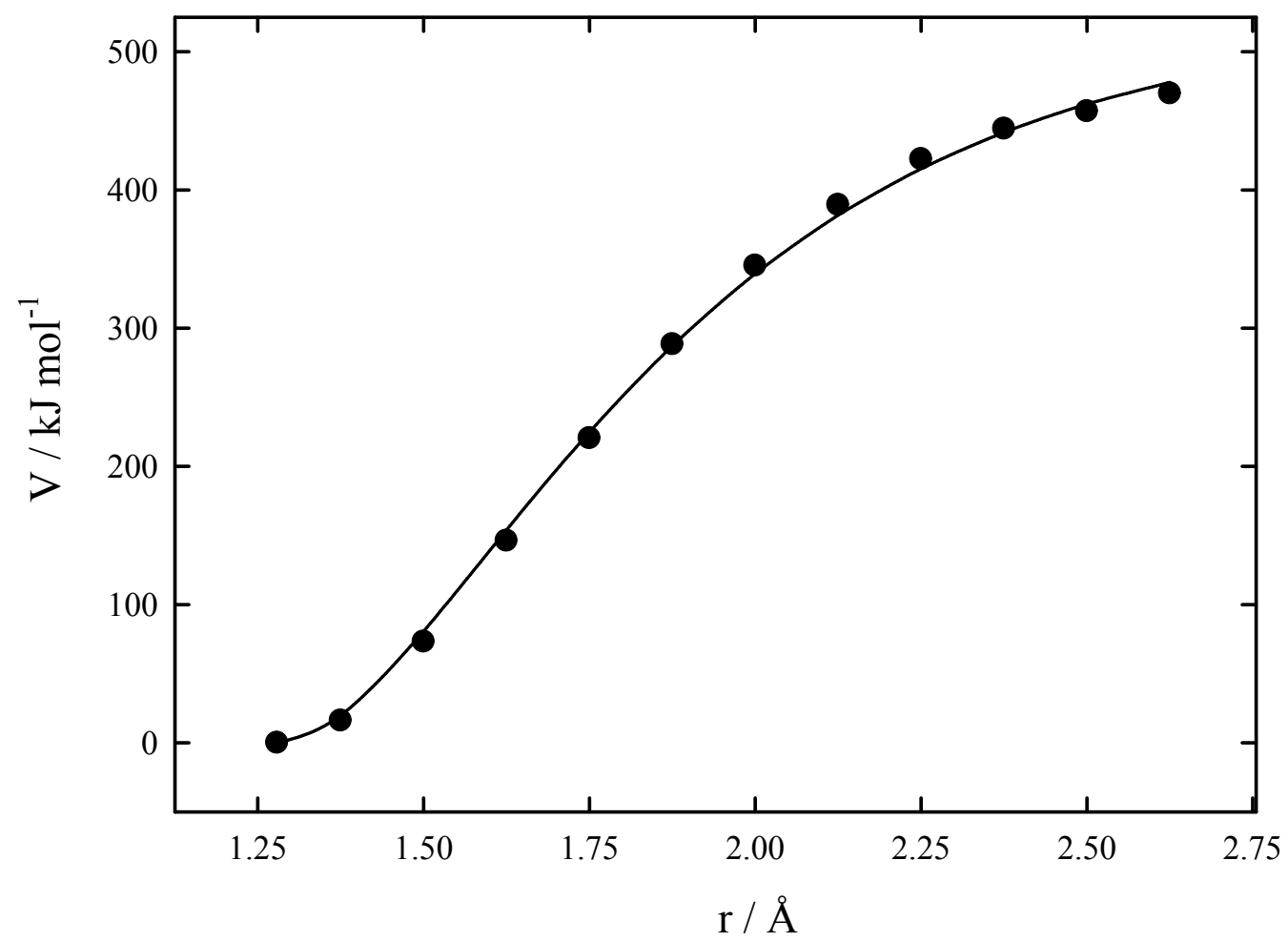

Fig. S9. Electronic potential for $\mathrm{C}_{2} \mathrm{~F}_{2} \rightarrow \mathrm{C}_{2} \mathrm{~F}+\mathrm{F}$ calculated at the $\mathrm{G} 4 / \mathrm{M} 06-2 \mathrm{X} / 6-311+\mathrm{G}(3 \mathrm{df})$ level. Solid line: fit with a Morse function $\left(\beta=2.24 \AA^{-1}\right)$. 


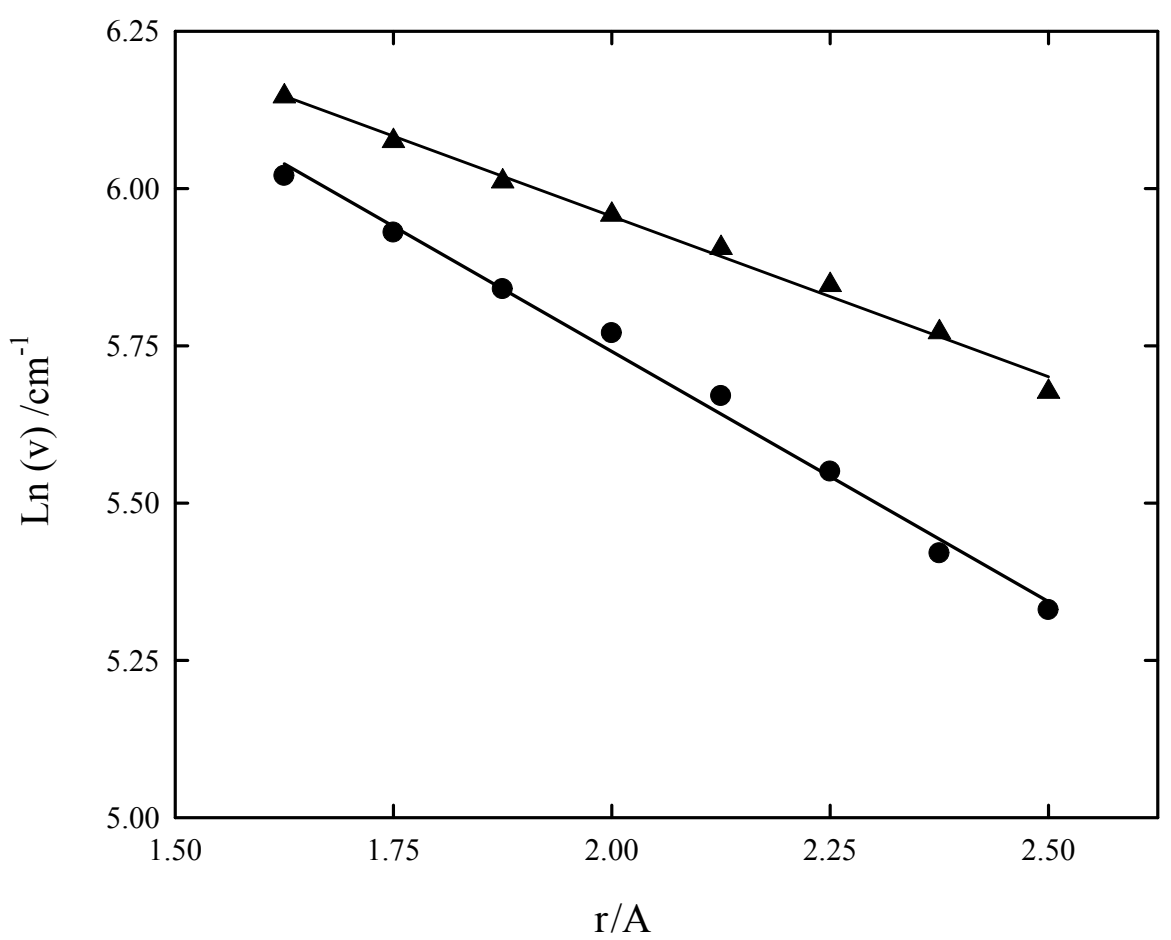

Fig. S10. Frequency of the transitional modes for $\mathrm{C}_{2} \mathrm{~F}_{2} \rightarrow \mathrm{C}_{2} \mathrm{~F}+\mathrm{F}$ calculated at the M06-2X/6$311+\mathrm{G}(3 \mathrm{df})$ level. Solid lines: fit with exponential decay functions with $\alpha=0.95 \AA^{-1}(\bullet)$ and $\alpha$ $=0.51 \AA^{-1}(\mathbf{\Delta})$. 


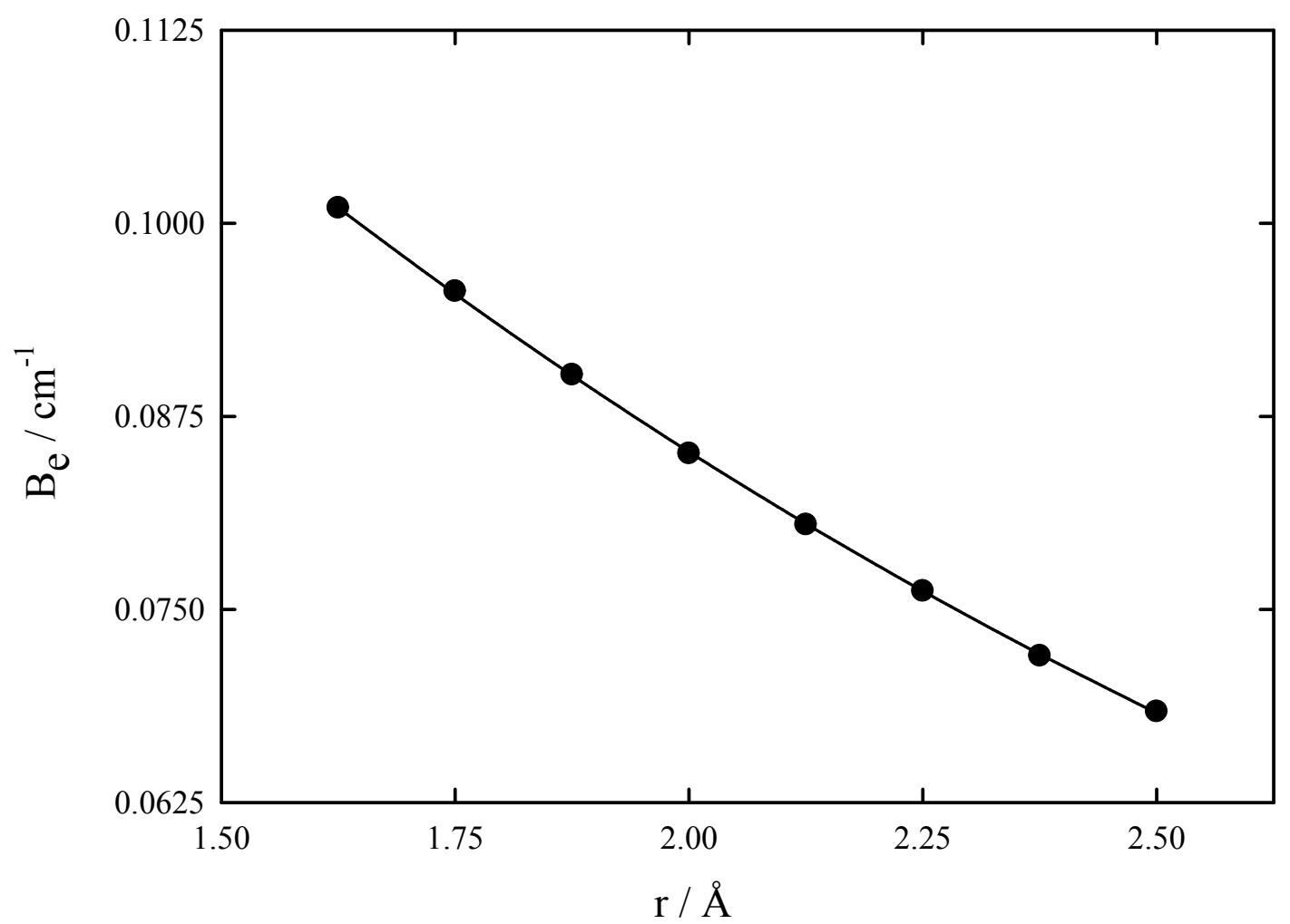

Fig. S11. Rotational constants of $\mathrm{C}_{2} \mathrm{~F}_{2}$ along the MEP, calculated at the M06-2X/6-311+G(3df) level. Solid line: fit with the function $(B+C) / 2=0.101 \mathrm{~cm}^{-1} /[1+0.4552(\mathrm{r}-1.625)+0.1055(\mathrm{r}$ $\left.-1.625)^{2}\right]$.

\section{Calculation of $k_{12, \infty}$}

Table S6. High-pressure rate constants for $\mathrm{C}_{2} \mathrm{~F}+\mathrm{F} \rightarrow \mathrm{C}_{2} \mathrm{~F}_{2}(-12)\left(\right.$ in $\left.^{3} \mathrm{~cm}^{3} \mathrm{~mol}^{-1} \mathrm{~s}^{-1}\right)$ and $\mathrm{C}_{2} \mathrm{~F}_{2} \rightarrow$ $\mathrm{C}_{2} \mathrm{~F}+\mathrm{F}(12)\left(\right.$ in s $\left.^{-1}\right)$. SACM/CT calculations with the parameters $v=1.16$ and $\mathrm{C}_{v}=5.12 \times 10^{-}$ ${ }^{3} \mathrm{~cm}^{-1}$, for details see ref. S4.

\begin{tabular}{|c|c|c|c|c|}
\hline$T / \mathrm{K}$ & $k_{-12, \infty} \mathrm{PST}$ & $\mathrm{f}_{\text {rigid }}$ & $k_{-12, \infty}$ & $k_{12, \infty}$ \\
\hline 1000 & $1.07 \times 10^{14}$ & 0.0927 & $9.88 \times 10^{12}$ & $1.41 \times 10^{-11}$ \\
\hline 2000 & $1.26 \times 10^{14}$ & 0.117 & $1.48 \times 10^{13}$ & $2.74 \times 10^{2}$ \\
\hline 3000 & $1.39 \times 10^{14}$ & 0.134 & $1.87 \times 10^{13}$ & $5.90 \times 10^{6}$ \\
\hline 4000 & $1.52 \times 10^{14}$ & 0.148 & $2.23 \times 10^{13}$ & $8.10 \times 10^{8}$ \\
\hline
\end{tabular}

$$
k_{-12, \infty}=5.17 \times 10^{15}(T / 3000 \mathrm{~K})^{-0.78} \exp (-61770 \mathrm{~K} / T) \mathrm{s}^{-1} \quad(2000-4000 \mathrm{~K})
$$




\section{Calculation of $k_{12,0}$}

Table S7. Low-pressure rate constants for $\mathrm{C}_{2} \mathrm{~F}_{2}(+\mathrm{Ar}) \rightarrow \mathrm{C}_{2} \mathrm{~F}+\mathrm{F}(+\mathrm{Ar})(12)\left(\mathrm{cm}^{3} \mathrm{~mol}^{-1} \mathrm{~s}^{-1}\right)$. The $\beta_{\mathrm{c}}$ values were calculated using a temperature independent value of $-<\Delta \mathrm{E} / \mathrm{hc}>=100 \mathrm{~cm}^{-1}$.

\begin{tabular}{|c|c|c|c|}
\hline$T / \mathrm{K}$ & $k_{12,0} \mathrm{SC}$ & $\beta_{\mathrm{c}}$ & $k_{12,0}$ \\
\hline 1000 & $3.46 \times 10^{-5}$ & 0.091 & $3.15 \times 10^{-6}$ \\
\hline 2000 & $1.53 \times 10^{7}$ & 0.047 & $7.19 \times 10^{5}$ \\
\hline 3000 & $3.26 \times 10^{10}$ & 0.030 & $9.78 \times 10^{8}$ \\
\hline 4000 & $8.12 \times 10^{11}$ & 0.020 & $1.62 \times 10^{10}$ \\
\hline
\end{tabular}

$k_{12,0}=[\mathrm{Ar}] 3.57 \times 10^{18}(T / 3000 \mathrm{~K})^{-9.36} \exp (-66055 \mathrm{~K} / T) \mathrm{cm}^{3} \mathrm{~mol}^{-1} \mathrm{~s}^{-1}(2000-4000 \mathrm{~K})$

\section{Calculation of falloff curves}

Falloff curves were calculated employing the model of ref. S5 with $\mathrm{F}_{\text {cent }}=\mathrm{F}_{\text {cent }}{ }^{\mathrm{SC}} \times \mathrm{F}_{\text {cent }}{ }^{\mathrm{WC}}=$ $\mathrm{F}_{\text {cent }} \mathrm{SC}_{\mathrm{X}} 0.64=0.14(2000 \mathrm{~K}), 0.13(3000 \mathrm{~K})$ and $0.13(4000 \mathrm{~K})$ values.

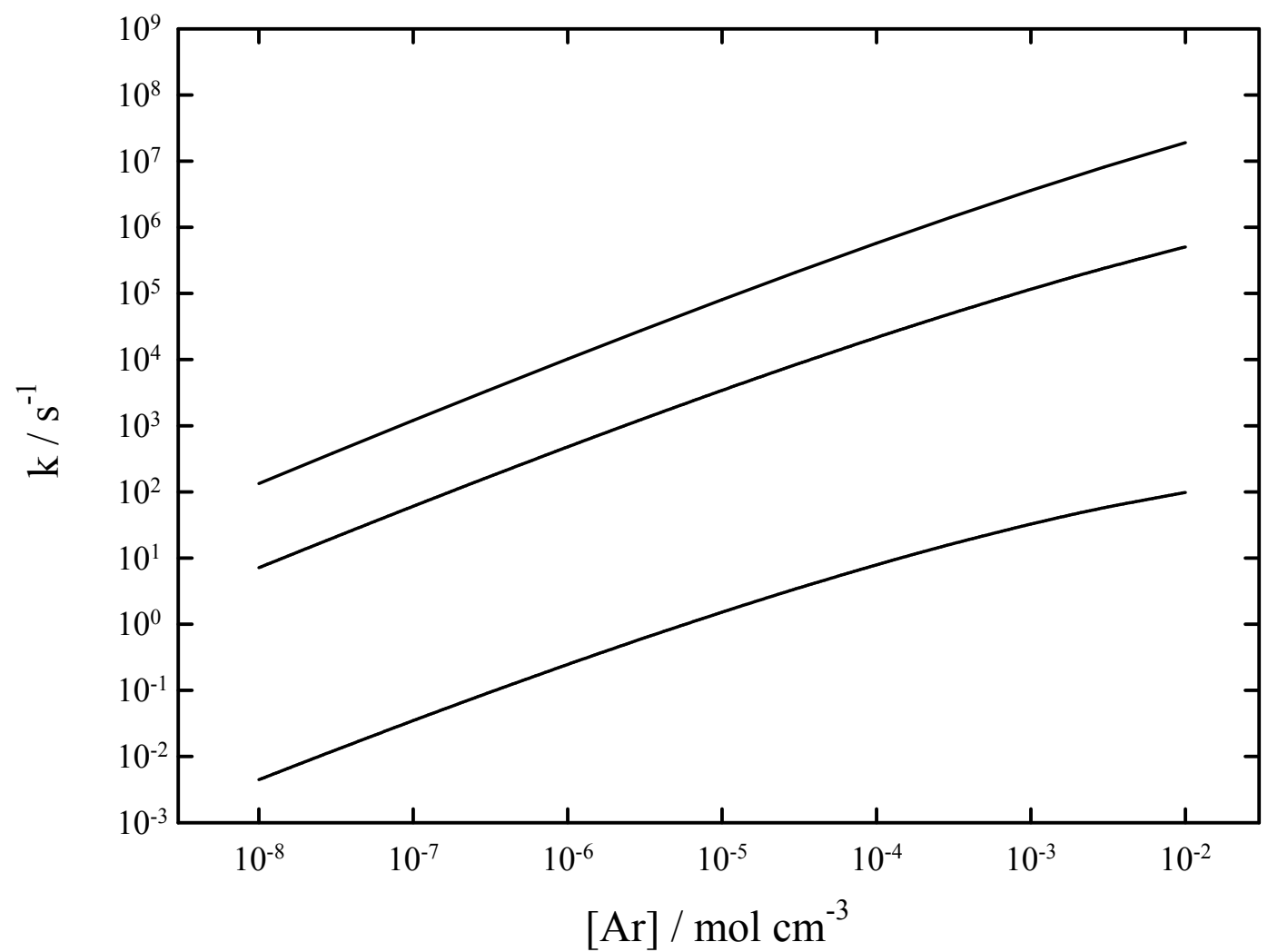

Fig. S12. Falloff curves for the reaction $\mathrm{C}_{2} \mathrm{~F}_{2}(+\mathrm{Ar}) \rightarrow \mathrm{C}_{2} \mathrm{~F}+\mathrm{F}(+\mathrm{Ar})$ (12). Calculations for 2000,3000 and $4000 \mathrm{~K}$ (from bottom to top). 


\section{Molecular parameters}

Bond dissociation enthalpy for $\mathrm{C}_{2} \mathrm{~F}_{2} \rightarrow \mathrm{C}_{2} \mathrm{~F}+\mathrm{F}$ : $511.7 \mathrm{~kJ} \mathrm{~mol}^{-1}$ (from G4//M06-2X/6$311+\mathrm{G}(3 \mathrm{df})$ calculations $)$.

$\mathrm{C}_{2} \mathrm{~F}_{2}$ vibrational frequencies: 287 (2), 358 (2), 800, 1369, $2550 \mathrm{~cm}^{-1}$ (from B3LYP/6$311+\mathrm{G}(3 \mathrm{df})$ calculations $)$.

$\mathrm{C}_{2} \mathrm{~F}_{2}$ rotational constants: $0.119 \mathrm{~cm}^{-1}$ (from B3LYP/6-311+G(3df) calculations).

$\mathrm{C}_{2} \mathrm{~F}$ vibrational frequency: 371, 1018, $1819 \mathrm{~cm}^{-1}$ (from B3LYP/6-311+G(3df) calculations).

$\mathrm{C}_{2} \mathrm{~F}$ rotational constants: $49.771,0.359,0.359 \mathrm{~cm}^{-11}$ (from B3LYP/6-311+G(3df) calculations).

\section{d. Falloff curves for the reaction $\mathrm{C}_{2} \mathrm{~F}_{2}(+\mathrm{Ar}) \rightarrow \mathrm{CF}+\mathrm{CF}(+\mathrm{Ar})$}

Details of calculations of the potentials and rate constants for the reaction (11) are found in the ESI of ref. S2. 
e. Falloff curves for the reaction $\mathrm{CF}+\mathrm{CF}(+\mathrm{Ar}) \rightarrow \mathrm{C}_{2} \mathrm{~F}_{2}(+\mathrm{Ar})$

Electronic potential along the MEP

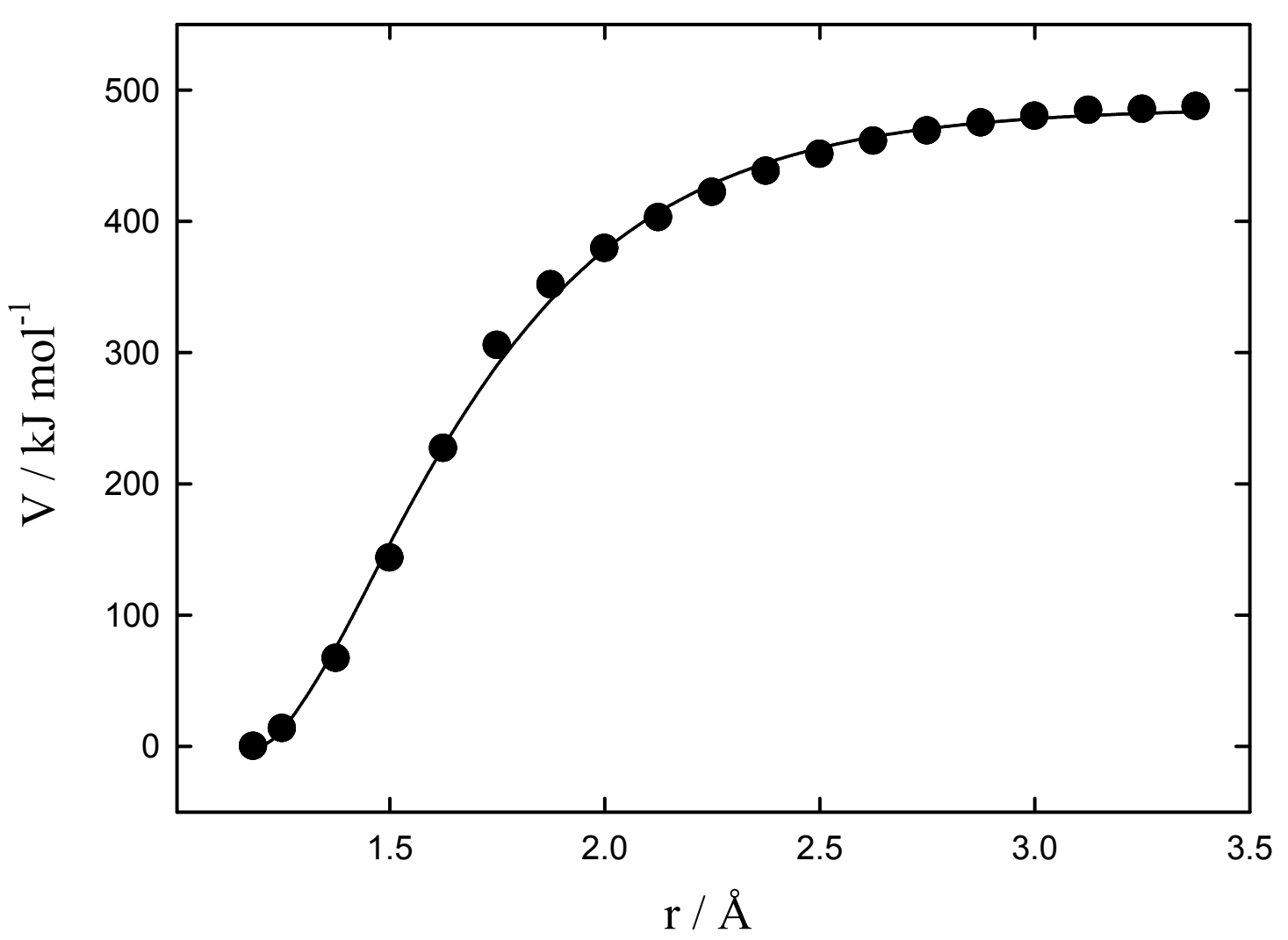

Fig. S13. Electronic potential for $\mathrm{CF}+\mathrm{CF} \rightarrow \mathrm{C}_{2} \mathrm{~F}_{2}$ calculated at the G4//B3LYP/6$311+\mathrm{G}(3 \mathrm{df})$ level. Solid line: fit with a Morse function $\left(\beta=2.61 \AA^{-1}\right)$. 


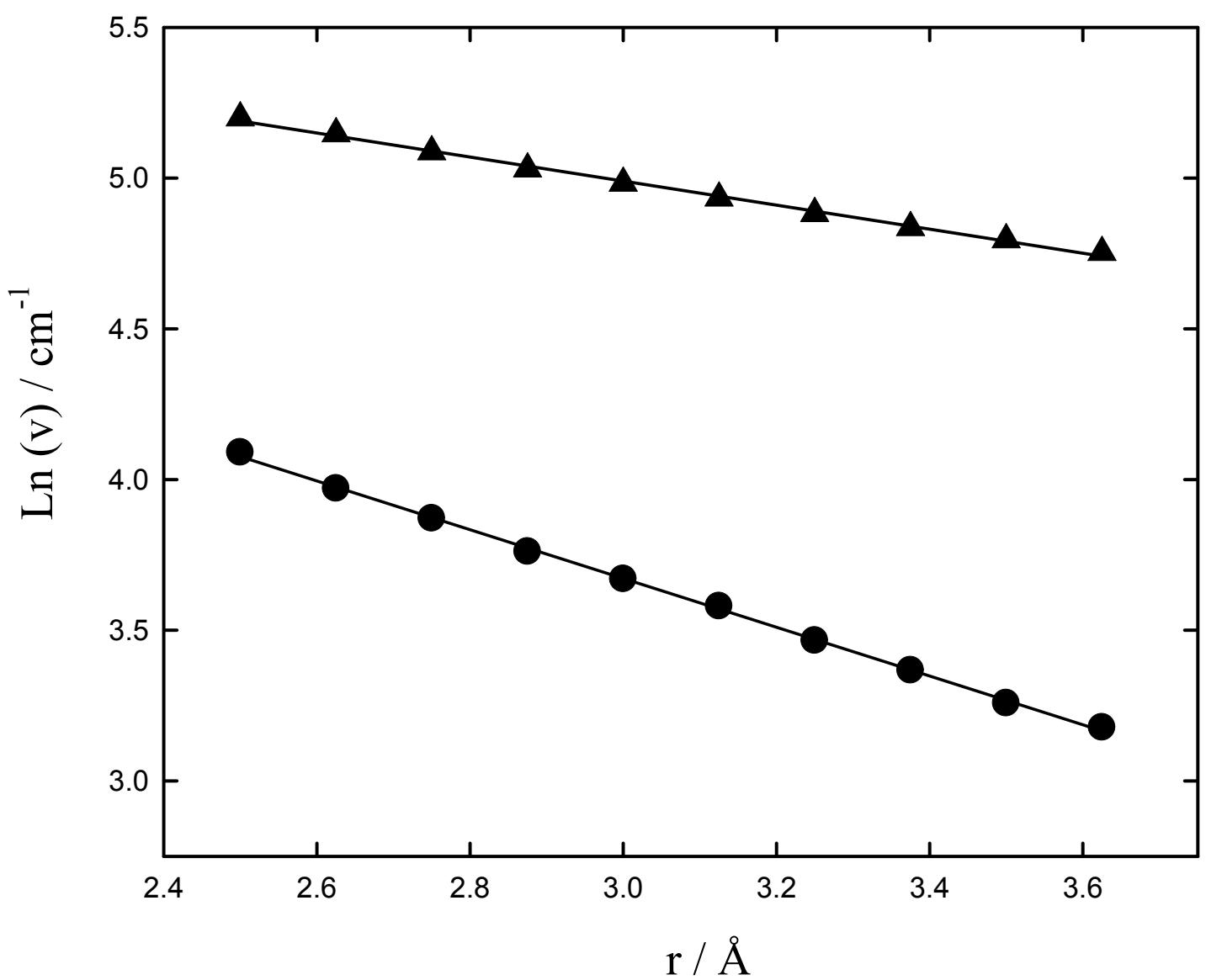

Fig. S14. Frequency of the transitional modes for $\mathrm{CF}+\mathrm{CF} \rightarrow \mathrm{C}_{2} \mathrm{~F}_{2}$ calculated at the B3LYP/6$311+\mathrm{G}(3 \mathrm{df})$ level. Solid lines: fit with exponential decay functions with $\alpha=0.80 \AA^{-1}(\bullet)$ and $\alpha$ $=0.41 \AA^{-1}(\mathbf{\Delta})$. 


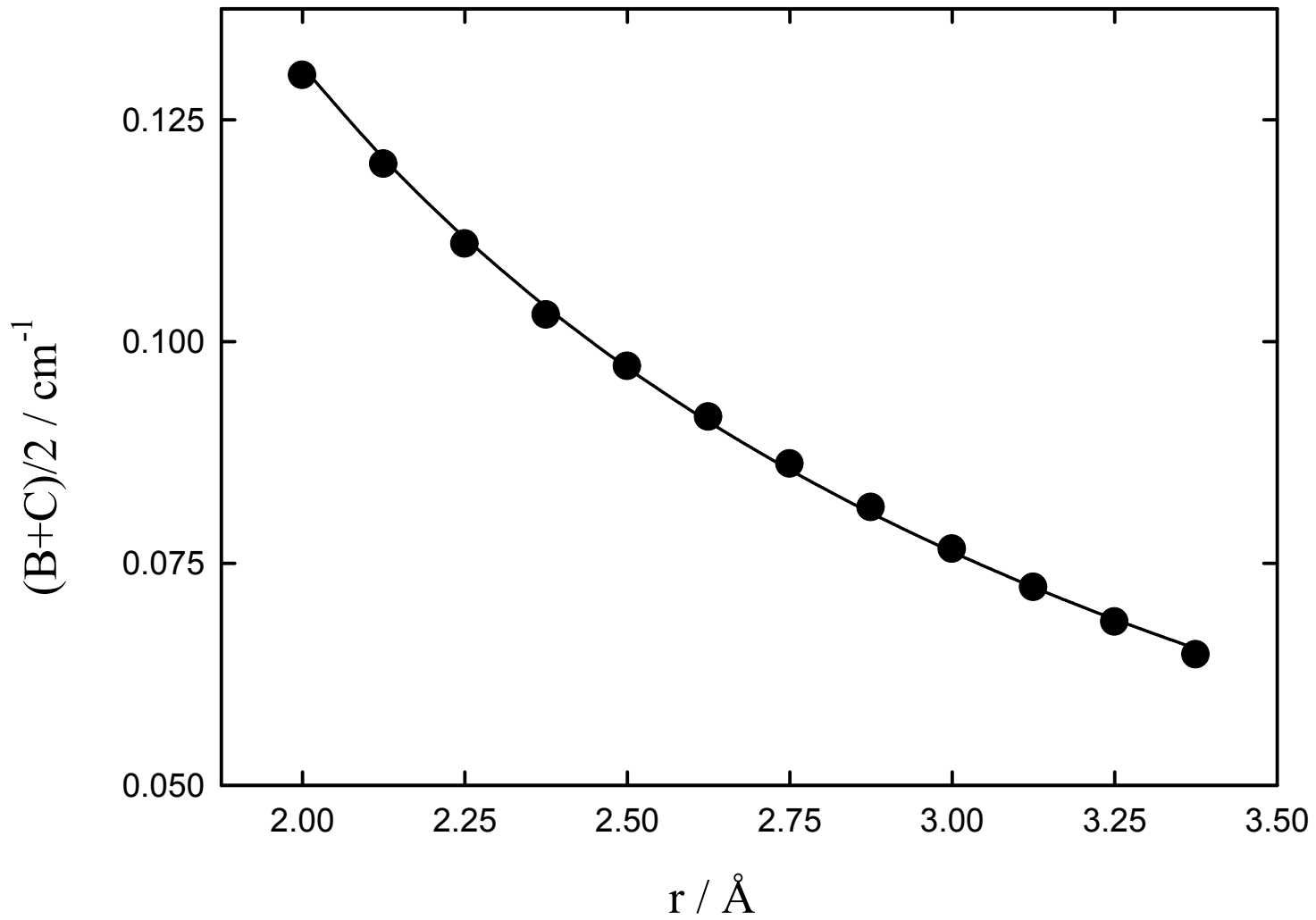

Fig. S15. Rotational constants of $\mathrm{C}_{2} \mathrm{~F}_{2}$ along the MEP, calculated at the B3LYP/6-311+G(3df) level. Solid line: fit with the function $(B+C) / 2=0.135 \mathrm{~cm}^{-1} /[1+0.6836(\mathrm{r}-2.0)+0.0335(\mathrm{r}-$ $\left.2.0)^{2}\right]$. 


\section{Calculation of $k_{10, \infty}$}

Table S8. High-pressure rate constants for $\mathrm{CF}+\mathrm{CF} \rightarrow \mathrm{C}_{2} \mathrm{~F}_{2}\left(\mathrm{in} \mathrm{cm}^{3} \mathrm{~mol}^{-1} \mathrm{~s}^{-1}\right)$ (10). SACM/CT calculations with the parameters $v=1.12$ and $\mathrm{C}_{v}=1.29 \times 10^{-2} \mathrm{~cm}^{-1}$, for details see ref. S4.

\begin{tabular}{|c|c|c|c|}
\hline$T / \mathrm{K}$ & $k_{10, \infty}{ }^{\text {PST }}$ & $\mathrm{f}_{\text {rigid }}$ & $k_{10, \infty}$ \\
\hline 750 & $1.29 \times 10^{14}$ & 0.0220 & $2.84 \times 10^{12}$ \\
\hline 1000 & $1.44 \times 10^{14}$ & 0.0299 & $4.31 \times 10^{12}$ \\
\hline 1500 & $1.68 \times 10^{14}$ & 0.0461 & $7.77 \times 10^{12}$ \\
\hline 2000 & $1.87 \times 10^{14}$ & 0.0625 & $1.17 \times 10^{13}$ \\
\hline
\end{tabular}

$$
k_{10, \infty}=4.31 \times 10^{12}(T / 1000 \mathrm{~K})^{1.45} \mathrm{~cm}^{3} \mathrm{~mol}^{-1} \mathrm{~s}^{-1}
$$

\section{Calculation of $\boldsymbol{k}_{10,0}$}

Table S9. Low-pressure rate constants for $\mathrm{CF}+\mathrm{CF}(+\mathrm{Ar}) \rightarrow \mathrm{C}_{2} \mathrm{~F}_{2}(+\mathrm{Ar})\left(\right.$ in $\left.\mathrm{cm}^{3} \mathrm{~mol}^{-1} \mathrm{~s}^{-1}\right)(10)$. The $\beta_{\mathrm{c}}$ values were calculated using a temperature independent value of $-<\Delta \mathrm{E} / \mathrm{hc}>=100 \mathrm{~cm}^{-1}$.

\begin{tabular}{|c|c|c|c|}
\hline$T / \mathrm{K}$ & $k_{10,0} \mathrm{SC}$ & $\beta_{\mathrm{c}}$ & $k_{10,0}$ \\
\hline 750 & $1.48 \times 10^{19}$ & 0.120 & $1.78 \times 10^{18}$ \\
\hline 1000 & $6.11 \times 10^{18}$ & 0.090 & $5.50 \times 10^{17}$ \\
\hline 1500 & $1.56 \times 10^{18}$ & 0.062 & $9.67 \times 10^{16}$ \\
\hline 2000 & $5.59 \times 10^{17}$ & 0.046 & $2.57 \times 10^{16}$ \\
\hline
\end{tabular}

$$
k_{10,0}=[\mathrm{Ar}] 1.37 \times 10^{18}(T / 1000 \mathrm{~K})^{-5.08} \exp (-905 \mathrm{~K} / T) \quad \mathrm{cm}^{3} \mathrm{~mol}^{-1} \mathrm{~s}^{-1}
$$




\section{Calculation of falloff curves}

Falloff curves were calculated employing the model of ref. S5 with $\mathrm{F}_{\text {cent }}=\mathrm{F}_{\text {cent }}{ }^{\mathrm{SC}} \times \mathrm{F}_{\text {cent }}{ }^{\mathrm{WC}}=$ $\mathrm{F}_{\text {cent }}{ }^{\mathrm{SC}} \mathrm{x} 0.64=0.26(750 \mathrm{~K}), 0.21(1000 \mathrm{~K}), 0.16(1500 \mathrm{~K})$ and $0.13(2000 \mathrm{~K})$ values.

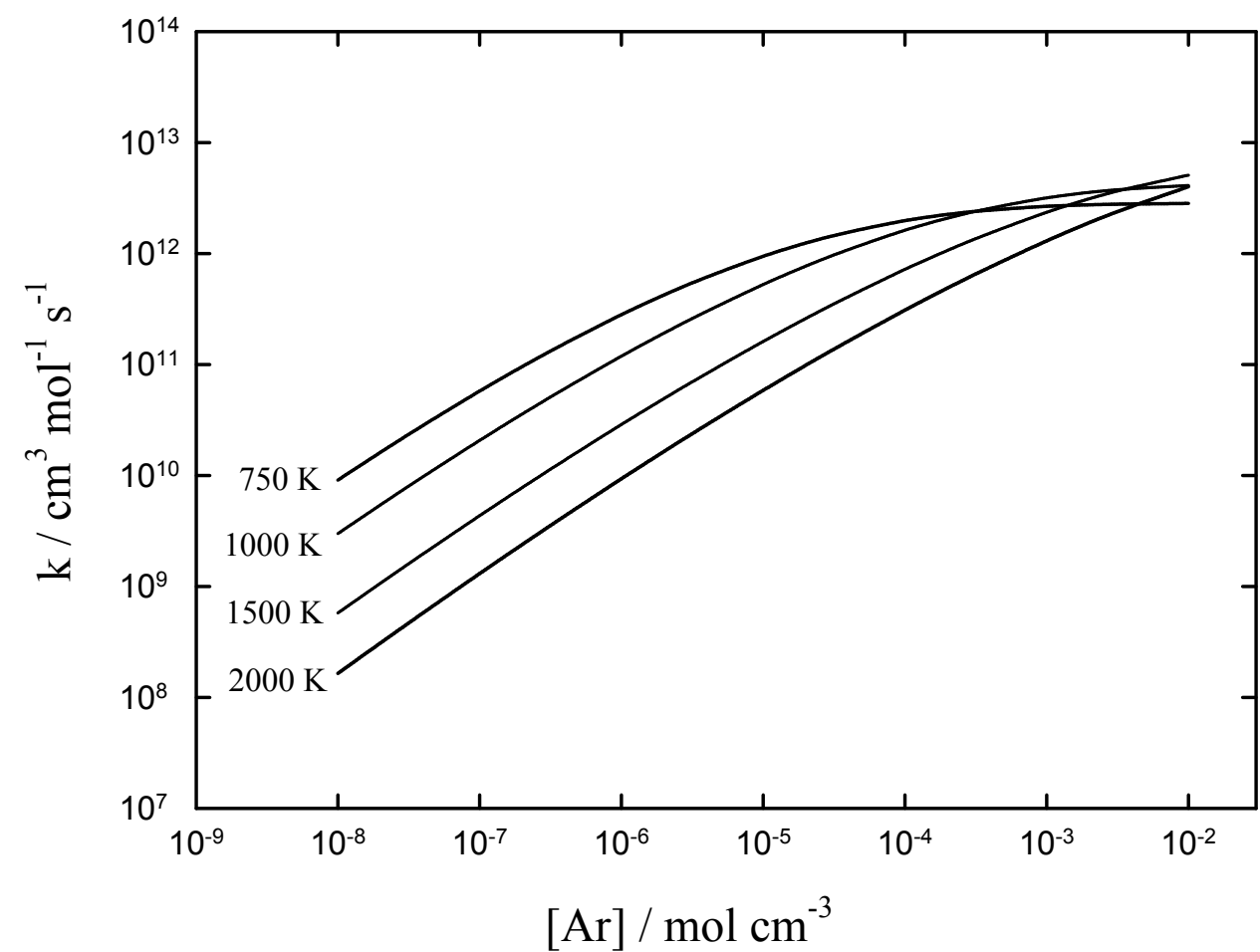

Fig. S16. Falloff curves for the reaction $\mathrm{CF}+\mathrm{CF}(+\mathrm{Ar}) \rightarrow \mathrm{C}_{2} \mathrm{~F}_{2}(+\mathrm{Ar})(10)$. Calculations for $2000,1500,1000$ and $750 \mathrm{~K}$ (from bottom to top).

\section{Molecular parameters}

Bond dissociation enthalpy for $\mathrm{CF}+\mathrm{CF}(+\mathrm{Ar}) \rightarrow \mathrm{C}_{2} \mathrm{~F}_{2}(+\mathrm{Ar}): 474.0 \mathrm{~kJ} \mathrm{~mol}^{-1}$.

$\mathrm{C}_{2} \mathrm{~F}_{2}$ vibrational frequencies: 287 (2), 358 (2), 800, 1369, $2550 \mathrm{~cm}^{-1}$ (from B3LYP/6$311+\mathrm{G}(3 \mathrm{df})$ calculations).

$\mathrm{C}_{2} \mathrm{~F}_{2}$ rotational constants: $0.119 \mathrm{~cm}^{-1}$ (from B3LYP/6-311+G(3df) calculations).

CF vibrational frequency: $1298 \mathrm{~cm}^{-1}$ (from B3LYP/6-311+G(3df) calculations).

CF rotational constants: $1.416 \mathrm{~cm}^{-11}$ (from B3LYP/6-311+G(3df) calculations). 


\section{f. Rate constants for the reaction $\mathrm{CF}+\mathrm{CF} \rightarrow \mathrm{C}_{2} \mathrm{~F}+\mathrm{F}$}

\begin{tabular}{|l|c|}
\hline \multicolumn{1}{|c|}{ Reaction } & $\Delta \mathrm{H}_{0}^{0} / \mathrm{kJ} \mathrm{mol}^{-1}$ \\
\hline $\mathrm{CF}+\mathrm{CF} \rightarrow \mathrm{C}_{2} \mathrm{~F}_{2}$ & -474.0 \\
\hline $\mathrm{CF}+\mathrm{CF} \rightarrow \mathrm{C}_{2} \mathrm{~F}+\mathrm{F}$ & 37.7 \\
\hline $\mathrm{C}_{2} \mathrm{~F} \rightarrow \mathrm{C}_{2}+\mathrm{F}$ & 444.8 \\
\hline $\mathrm{C}_{2} \mathrm{~F} \rightarrow \mathrm{CF}+\mathrm{C}$ & 511.3 \\
\hline $\mathrm{C}_{2} \mathrm{~F}+\mathrm{C}_{2} \mathrm{~F} \rightarrow \mathrm{FC} \equiv \mathrm{C}-\mathrm{C} \equiv \mathrm{CF}$ & -655.8 \\
\hline
\end{tabular}

Table S10. Reaction enthalpies at the G4//B3LYP/6-311+G(3df) level (at $0 \mathrm{~K}$ ).

The reaction $\mathrm{CF}+\mathrm{CF} \rightarrow \mathrm{C}_{2} \mathrm{~F}+\mathrm{F}$ (reaction (7)) apparently proceeds via intermediate formation of $\mathrm{C}_{2} \mathrm{~F}_{2}$ without electronic energy barrier higher than the endothermicity. Its rate constant then is estimated by multiplying the high pressure rate constant $k_{10, \infty}=4.31 \times 10^{12}$ $(\mathrm{T} / 1000 \mathrm{~K})^{1.45} \mathrm{~cm}^{3} \mathrm{~mol}^{-1} \mathrm{~s}^{-1}$ (from SACM/CT calculations for the reaction $\mathrm{CF}+\mathrm{CF} \rightarrow$ $\mathrm{C}_{2} \mathrm{~F}_{2}$ (reaction (10)) with the equilibrium constant $K_{\mathrm{c}}=k_{10, \infty} / k_{7}=\left(\left[\mathrm{C}_{2} \mathrm{~F}\right][\mathrm{F}] /[\mathrm{CF}]^{2}\right)_{\text {eq }}=0.22$ $(T / 1000 \mathrm{~K}) \exp (-4355 \mathrm{~K} / T)$. This leads to $k_{7}=2.2 \times 10^{12}(T / 1000 \mathrm{~K})^{1.0} \exp (-4400 \mathrm{~K} / T) \mathrm{cm}^{3}$ $\mathrm{mol}^{-1} \mathrm{~s}^{-1}$ (for more details, see the ESI of ref. S2). Reaction (7) competes with the reaction $\mathrm{CF}+\mathrm{CF}(+\mathrm{Ar}) \rightarrow \mathrm{C}_{2} \mathrm{~F}_{2}(+\mathrm{Ar})$. According to the falloffcurves of the latter reaction (see above in part $\mathbf{e}$ ), for temperatures above $2000 \mathrm{~K}$ and [Ar] below $10^{-4} \mathrm{~mol} \mathrm{~cm}^{-3}$, reaction (7) dominates. 


\section{References}

(S1) Cobos, C. J.; Knight, G.; Sölter, L.; Tellbach, E.; Troe, J., J. Phys. Chem. A, 2017, 121, 7827.

(S2) Cobos, C. J.; Sölter, L.; Tellbach, E.; Troe, J., Phys. Chem. Chem. Phys. 2021, 23, 4394.

(S3) Sharpe, S.; Hartnett, B.; Sethi, H. S.; Sethi, D. S., J. Photochem. 1987, $38,1$.

(S4) Cobos, C. J.; Knight, G.; Sölter, L.; Tellbach, E.; Troe, J., J. Phys. Chem. A 2020, 124, 1235.

(S5) Troe, J.; Ushakov, V. G., Z. Phys. Chem.2013, 228, 1.

(S6) Chemical Kinetics and Photochemical Data for Use in Atmospheric Studies, JPL evaluation number 19 (https://jpldataeval.jpl.nasa.gov/pdf/NASA-JPL).

(S7) Burkholder, J. B. Howard, C. J. Hamilton, P. A., J. Mol. Spectrosc. 1988, 127, 362.

(S8) Qian, H.-B.; Davies, P. B., J. Mol. Spectrosc. 1995, 169, 201.

(S9) Marquez, L. Demaison, J. Boggs, J. E., J. Phys. Chem. A1999, 103, 7632. 